1 THE USE AND IMPACT OF 12-LEAD ELECTROCARDIOGRAMS IN ACUTE STROKE PATIENTS: A SYSTEMATIC REVIEW

S Munro*, T Quinn, D Cooke, M Joy. University of Surrey, Guildford, UK

\subsection{6/bmjopen-2017-EMSabstracts.1}

Aim Stroke is a leading cause of mortality and disability across the globe. Emergency Medical Services assess and transport a large number of these patients in the prehospital setting. Guidelines for UK ambulance services recommend recording a 12-lead electrocardiogram in the prehospital environment, providing this does not add to significant delay in transporting the patient to hospital; however, this recommendation is not based on any evidence.

Methods A systematic review was conducted to search and synthesise the literature surrounding the use of prehospital electrocardiograms in acute stroke patients, focusing on the prevalence of abnormalities and their association with prognosis and outcome. Online databases, references from selected articles and hand searches were made to identify eligible studies. Two authors independently reviewed the studies to ensure eligibility criteria were met. Main outcomes were presence of abnormality on electrocardiogram, mortality and disability. No studies set in the prehospital environment were found by the search; therefore the eligibility criteria were widened to include hospital-based studies. A total of 18 studies were subsequently included in the review.

Results Although the prevalence of electrocardiogram abnormalities appears common in hospitalised patients, their prognostic impact on mortality, disability and other adverse outcomes is conflicting amongst the literature. There is a lack of research surrounding the use of prehospital electrocardiogram in acute stroke patients.

Conclusion Future studies should be based in the prehospital environment and should investigate whether undertaking an electrocardiogram in the prehospital setting affects clinical management decisions or has an association with mortality or morbidity.

Conflict of interest None declared

Funding This work was supported by School of Health Sciences PhD bursary - University of Surrey, and South East Coast Ambulance Service NHS Foundation Trust.

\section{DOES CURRENT PRE-HOSPITAL ANALGESIA EFFECTIVELY REDUCE PAIN IN CHILDREN CAUSED BY TRAUMA WITHIN A UK AMBULANCE SERVICE: A SERVICE EVALUATION}

G Whitley* ${ }^{*}$ F Bath-Hextall. University of Nottingham

10.1136/bmjopen-2017-EMSabstracts.2

Aim Analgesic treatment of pre-hospital injured children is viewed as 'suboptimal' with few receiving analgesia. The aim of this study was to explore current analgesia given to traumatically injured children in the pre-hospital setting and examine whether a clinically meaningful reduction in pain was achieved.

Methods We evaluated electronic patient report forms over a two year period (2013 and 2014) within a UK ambulance service NHS trust. All traumatically injured children within the age range of 1 to 17 with a clinical impression of a fracture, dislocation, wound or burn were included. Patients with a Glasgow Coma Scale of $<15$ were excluded. The outcome measure was a reduction in numeric pain rating scale or Wong and Baker faces of $\geq 2$ out of 10 .

Results Of the evaluable patients $(n=11,317)$, 90.8\% had a documented pain score, or a reason why a pain score could not be documented. For patients reporting pain $(n=7,483)$, $51.6 \%(n=3,861)$ received analgesia, 9.6\% $(n=717)$ received no analgesia but did receive alternative treatment and $38.8 \%$ $(n=2,905)$ received no analgesia and no alternative treatment. Morphine sulphate IV, oral morphine, Entonox, paracetamol suspension and poly-analgesia all achieved a clinically meaningful median reduction in pain score.

Conclusion Analgesia administered to traumatically injured children in the pre-hospital setting within this UK ambulance service NHS trust does produce clinically meaningful reductions in pain. The concern is that a large number of patients received no analgesia or alternative treatment. There is a real need to identify barriers to analgesia administration in this patient group.

Conflict of interest None declared.

Funding None declared.

\section{CHOKING CHILDREN - SHOULD WE BE SCARED?}

H Frederiksen Højgaard*, D Köhler. Lillebælt Hospital, Denmark

\subsection{6/bmjopen-2017-EMSabstracts.3}

Aim Choking in children is a feared condition, which potentially can deteriorate to hypoxic cardiac arrest and death. Fast recognition and immediate initiation of basic life support (BLS)1, primary back blows and abdominal thrusts given by bystanders and secondary activation of the Emergency Medical System (EMS) are vital for the survival.

Methods The Region of Southern Denmark has a tiered response with prehospital emergency physicians to supplement the ambulance service. EMS physicians register all contacts in a database. We reviewed the database for all children below the age of 5 years who suffered from choking in 2009 to 2014.

Results From 70.289 total contacts 4.857 contacts was with children less than 5 years. In this group 98 children suffered from choking. In $78 \%$ BLS had resolved the obstructing foreign body before arrival of EMS services. Seventeen children had successful treatment with BLS by ambulance personal. Five children required advanced airway management and one child required cardiopulmonary resuscitation. No deaths were registered. None of the children with near fatal choking had any comorbidity prior to incident.

Conclusion Choking in children is an uncommon emergency with only $0,14 \%$ of all contacts. We found like others 2 that BLS solves most cases before arrival of EMS. This demonstrates the importance of immediate initiation of BLS and keeping the chain of survival as a concept. Death to choking is rare among small children. Near fatal choking resolves with skills and routine in advanced airway management and advanced life support.

\section{REFERENCES}

1. Maconochie IK, Bingham R, Eich C, López-Herce J4, Rodríguez-Núñez A, Rajka T, Van de Voorde P, Zideman DA, Biarent D: European Resuscitation Council Guidelines for Resuscitation 2015: Section 6. Paediatric life support. Resuscitation. 2015 Oct;95:223-48. 
2. Vilke GM, Smith AM, Ray LU, Steen PJ, Murrin PA, Chan TC: Airway obstruction in children aged less than 5 years: the prehospital experience. Prehosp Emerg Care 2004, 8:196-199.

Conflict of interest None declared. Funding None declared.

\section{REVISING EMS DISPATCH PROCEDURES TO MANAGE GROWING DEMAND IN VICTORIA, AUSTRALIA}

${ }^{1} \mathrm{E}$ Andrew* ${ }^{1,2}{ }^{2}$ Karen Smith. ${ }^{1}$ Ambulance Victoria; ${ }^{2}$ Monash University

\subsection{6/bmjopen-2017-EMSabstracts.4}

Aim The Advanced Medical Priority Dispatch (AMPDS) system is used to triage emergency medical calls in Victoria, Australia. However, the level of response assigned to each AMPDS Event Type (ET), which may include triage away from an emergency medical service (EMS) attendance, is determined by Ambulance Victoria. This study aimed to increase the proportion of low-acuity calls diverted to secondary triage and onto an alternative service provider (ASP), ultimately reducing EMS demand.

Methods A review of the level of EMS response assigned to AMPDS ET was conducted using research, audit and clinical expertise. Existing 'time-critical' and 'acute but not time-critical' ETs were assessed for suitability against the assigned level of EMS response and existing dispatch rules. An analysis of events occurring pre-reform (8 Feb-8 May 2016) and postreform (9 May-8 Aug 2016) was conducted.

Results A total of 105 'time-critical' ETs were assessed as suitable for downgrade to an 'acute' response, while 221 'acute' and 'time-critical' ETs were deemed suitable for diversion to secondary triage. The changes were implemented using staged approach, commencing in October 2015. The proportion of cases receiving a 'time-critical' EMS response decreased from $55.1 \%$ pre-reform to $48.9 \%$ post-reform $(\mathrm{p}<0.001)$. The proportion of emergency calls avoiding EMS dispatch increased from $8.8 \%$ pre-reform to $14.1 \%$ post-reform $(p<0.001)$. Of the cases diverted to secondary triage post-reform, $32.4 \%$ were referred to an ASP, $30.2 \%$ were referred to a non-emergency transport service and $41.8 \%$ were returned for EMS dispatch.

Conclusion This study provides a sound methodological approach for revising EMS dispatch protocols. Ongoing monitoring of the dispatch changes aims to identify areas that may benefit from further optimisation.

Conflict of interest None declared.

Funding None declared.

\section{MENTAL HEALTH-RELATED PRESENTATIONS TO EMERGENCY MEDICAL SERVICES IN VICTORIA, AUSTRALIA}

${ }^{1} \mathrm{E}$ Andrew*, ${ }^{*} \mathrm{R}$ Roggenkamp, ${ }^{1,2} \mathrm{Z}$ Nehme, ${ }^{1,2} \mathrm{~S}$ Cox, ${ }^{1,2} \mathrm{~K}$ Smith. ${ }^{1}$ Ambulance Victoria; ${ }^{2}$ Monash University

\subsection{6/bmjopen-2017-EMSabstracts.5}

Aim Recent increases in demand for mental health services have not been matched with community resources, resulting in increased demand on emergency medical services (EMS). We sought to describe the clinical profile, demand patterns and care provided to mental health patients accessing EMS in Victoria, Australia.

Methods We conducted a retrospective observational study of cases occurring between January and December 2015. Computer Aided Dispatch and electronic patient care record data were extracted from the Ambulance Victoria data warehouse. Characteristics of mental health presentations were described using descriptive statistics.

Results Of the total 504,676 EMS attendances, 48041 (9.5\%) were mental health-related. Additionally, 4708 mental healthrelated secondary triages were included, representing $6.6 \%$ of the Victorian secondary triage caseload. Emergency mental health patients were younger and more often female than other patients attended by EMS $(p<0.001)$. Most mental health patients were transported to hospital (74.4\%), however paramedics provided treatment to significantly fewer mental health patients than other emergency patients $(12.4 \%$ vs. $50.3 \%, \mathrm{p}<0.001)$. In mental health patients $\leq 15$ years, the most common presentations involved social/emotional issues, while for patients aged $\geq 65$ years, the most common presentation was anxiety. In patients undergoing secondary triage, $52.5 \%$ were frequent callers or anxiety presentations. A total of $27.7 \%$ of triaged patients were referred to an alternative service, while $24.6 \%$ were managed as per a care plan.

Conclusion For mental health-related cases, EMS were predominantly utilised as a transport mechanism rather than for active medical intervention. The role of alternative services in provision of care for mental health patients requires further investigation.

Conflict of interest None declared.

Funding None declared.

\section{CHARACTERISTICS OF THUNDERSTORM ASTHMA EMS ATTENDANCES IN VICTORIA, AUSTRALIA}

${ }^{1} \mathrm{E}$ Andrew* ${ }^{*}{ }^{1,2} \mathrm{Z}$ Nehme, ${ }^{1,2,3} \mathrm{~S}$ Bernard, ${ }^{1,2} \mathrm{~K}$ Smith. ${ }^{1}$ Ambulance Victoria; ${ }^{2}$ Monash University; ${ }^{3}$ The Alfred Hospital

\subsection{6/bmjopen-2017-EMSabstracts.6}

Aim Thunderstorm Asthma (TA) occurs when a dangerous mix of pollen and severe weather trigger acute respiratory distress symptoms in people with allergic rhinitis and asthma. We sought to describe the characteristics of patients attended by emergency medical services (EMS) during the largest global epidemic of TA which occurred in Melbourne on 21/11/2016. Methods A retrospective observational study of electronic EMS patient care records was conducted for all cases occurring during TA, between $1800 \mathrm{hrs}$ on $21 / 11 / 2016$ and 2359 hours on 22/11/2016 (30 hours). Results were compared with a standard comparator period defined as the seven days prior to the event $(14 / 11 / 2016$ to $20 / 11 / 2016)$.

Results EMS responded to 3631 cases during the TA event, compared with an average of 2419 cases per 30 hours during the comparator period. During TA, the final paramedic diagnosis was acute respiratory distress in $28.3 \%$ of patients (Asthma $=18.0 \%$, Shortness of Breath $=10.3 \%$ ), compared with $3.6 \%$ of patients during the comparator period (Asthma $=0.6 \%$, Shortness of Breath $=3.0 \%, \mathrm{p}<0.001$ ). Whilst there was an absolute increase in the number of time-critical cases, the proportion of acute respiratory illness patients considered time-critical after initial paramedic assessment remained stable between the two periods $(42.0 \%$ vs. $43.5 \%, \mathrm{p}=0.6)$. 